\title{
Measurement of tissue optical properties by the use of oblique-incidence optical fiber reflectometry
}

\author{
Shao-Pow Lin, Lihong Wang, Steven L. Jacques, and Frank K. Tittel
}

\begin{abstract}
Fiber-optic-based oblique-incidence reflectometry is a simple and accurate method for measuring the absorption and reduced scattering coefficients $\mu_{a}$ and $\mu_{s}^{\prime}$ of semi-infinite turbid media. Obliquely incident light produces a spatial distribution of diffuse reflectance that is not centered about the point of light entry. The amount of shift in the center of diffuse reflectance is directly related to the medium's diffusion length $D$. We developed a fiber-optic probe to deliver light obliquely and sample the relative profile of diffuse reflectance. Measurement in absolute units is not necessary. From the profile, it was possible to measure $D$, perform a curve fit for the effective attenuation coefficient $\mu_{\mathrm{eff}}$, and then calculate $\mu_{a}$ and $\mu_{s}^{\prime}$. This method was verified with Monte Carlo simulations and tested on tissue phantoms. Our measurements of $D$ and $\mu_{\text {eff }}$ had an accuracy of approximately $5 \%$, thus giving us $10 \%$ and $5 \%$ accuracy for $\mu_{a}$ and $\mu_{s}^{\prime}$, respectively. (C) 1997 Optical Society of America

Key words: Tissue optics, fiber optics, biomedical optics, turbid media, scattering media, optical properties, reflectometry, oblique incidence.
\end{abstract}

\section{Introduction}

In this paper we present continued work on the development of oblique-incidence reflectometry as originally conceived by Wang and Jacques. ${ }^{1}$ The original paper considered measurement of only the reduced scattering coefficient of turbid media. Our current work describes a method for determining both absorption and reduced scattering and presents results with a prototype of a fiber-optic probe. Section 2 explains the theory behind the method, which has been verified with Monte Carlo simulations and tested on tissue phantoms. Section 3 contains results, and Section 4 gives an in-depth discussion.

The measurement of optical properties, namely, absorption coefficient $\mu_{a}$ and reduced scattering coefficient $\mu_{s}^{\prime}$, of biological tissues remains a central

S.-P. Lin, S. L. Jacques, and F. K. Tittel are with the Department of Electrical and Computer Engineering, Rice University, P.O. Box 1892, Houston, Texas 77251. S.-P. Lin and S. L. Jacques are with the Laser Biology Research Laboratory, Box 17, University of Texas M. D. Anderson Cancer Center, 1515 Holcombe Boulevard, Houston, Texas 77030. L. Wang, the corresponding author, is with the Bioengineering Program, Texas A\&M University, 234C Zachry Engineering Center, College Station, Texas 77843.

Received 4 March 1996; revised manuscript received 17 September 1996.

0003-6935/97/010136-08 $\$ 10.00 / 0$

(C) 1997 Optical Society of America problem in the field of biomedical optics. Knowledge of these parameters is important in both therapeutic and diagnostic applications of light in medicine. For example, optical properties are necessary to make accurate assessments of local fluence during irradiation procedures such as photodynamic therapy, photocoagulation, and tissue ablation. Also, in addition to having a profound impact on in vivo diagnostics such as optical imaging and fluorescence spectroscopy, the optical properties themselves can potentially be used to provide metabolic information and diagnose diseases.

To date, a number of methods have been developed to measure tissue optical properties. Probably the most common technique is the integrating-sphere measurement, but this technique requires the removal of a thin slice of tissue for both reflection and transmission measurements. ${ }^{2}$ A second technique is normal-incidence video reflectometry. One variation (which is based on diffusion theory) of this technique provides a noninvasive way to determine optical properties, but requires a measurement of both the spatial distribution of diffuse reflectance and the total diffuse reflectance. ${ }^{3}$ Another variation involves training a neural network with Monte Carlosimulated reflectance data and measurement of diffuse reflectance in absolute units. ${ }^{4}$ The latter technique has also been utilized with a normalincidence fiber-optic probe. ${ }^{5}$ There are several 
drawbacks to these techniques. Calibration to absolute units is a sensitive procedure and hence is not ideal for a clinical setting. With video reflectometry, there is no convenient way to make multiwavelength measurements with a white-light source simultaneously. In addition, the application of these methods to multiwavelength measurements requires a full calibration to the spectral output and responsivity of the source and the detector. Finally, time-resolved and frequency-domain techniques require instrumentation that may not be cost effective for nonresearch applications. In short, noninvasive, inexpensive, and real-time measurement of tissue optical properties has remained a challenge.

\section{Materials and Methods}

\section{A. Theory}

When light enters a semi-infinite tissue, it will generally scatter a number of times before either being absorbed or escaping the tissue surface at a point other than its point of entry. The multiply scattered light that escapes is called diffuse reflectance. Monte Carlo simulations of light transport accurately reproduce spatially resolved diffuse reflectance data, but iterative algorithms that take advantage of this accuracy to determine optical properties are impractical because the simulations are computationally intensive and relatively slow. 6,7 In this paper we utilize a simple two-source diffusion theory model of spatially resolved, steady-state diffuse reflectance. ${ }^{8}$ This diffusion theory model does not accurately model near diffuse reflectance, that is, reflectance that falls in the range of 1-2 transport mean free paths $\left(\mathrm{mfp}^{\prime}\right)$ of the source, but its simplicity makes up for this drawback by providing an efficient and conceptually clear means of reconstructing optical properties. Moreover, this disadvantage can be avoided as long as no attempt is made to fit data too close to the source to the diffusion theory model [Fig. 1(a)]. From this point on, any reference to diffuse reflectance specifically excludes near-diffuse reflectance.

Because it is easier to model isotropic scattering than anisotropic scattering, the reduced or transport scattering coefficient $\mu_{s}^{\prime}$ is introduced as the equivalent isotropic scattering coefficient of an otherwise anisotropically scattering medium. $\mu_{s}^{\prime}=\mu_{s}(1-g)$, where $\mu_{s}$ is the scattering coefficient and $g$ is the average cosine of the scattering angle. The transport mean free path is defined as

$$
\operatorname{mfp}^{\prime}=1 /\left(\mu_{a}+\mu_{s}^{\prime}\right)
$$

The spatial distribution of diffuse reflectance of normally incident light has previously been modeled by Farrell et al., ${ }^{8}$ who used two isotropic point sources, one positive source located $1 \mathrm{mfp}^{\prime}$ below the tissue surface and one negative image source above the tissue surface. The positive source represents a single scatter source in the tissue, and the height in $z$ of the image source depends on the boundary condition.

With oblique incidence, we propose that the buried
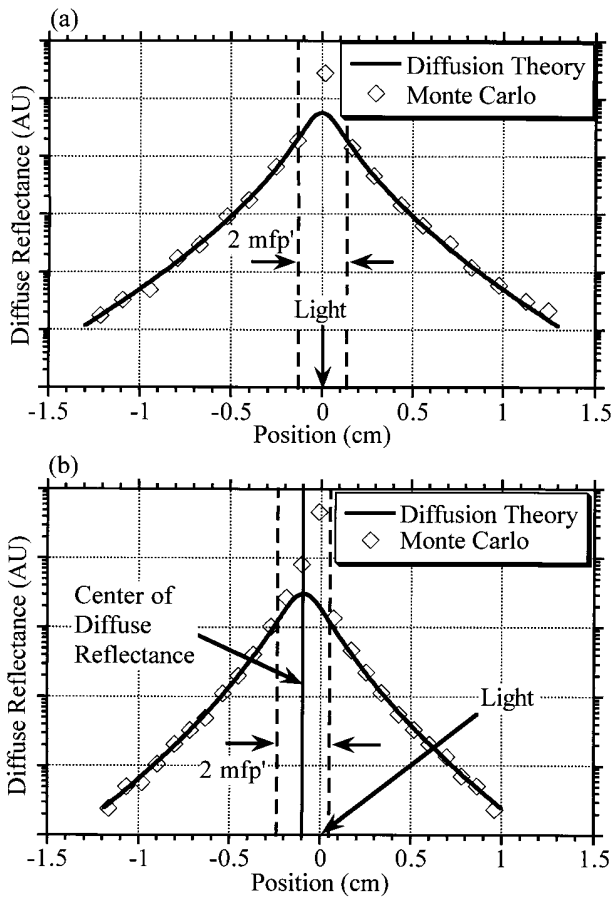

Fig. 1. Diffuse reflectance from a semi-infinite tissue as modeled by Monte Carlo simulation versus the two-source diffusion theory model. $\mu_{a}=0.4 \mathrm{~cm}^{-1}, \mu_{s}^{\prime}=8 \mathrm{~cm}^{-1}$, and $1 \mathrm{mfp}^{\prime}=0.123 \mathrm{~cm}$. (a) Normal incidence, (b) oblique incidence. For both (a) and (b), the two curves agree outside 1-2 $\mathrm{mfp}^{\prime}$ from the center of diffuse reflectance, which coincides with the light entry point in (a) but not in (b).

source should be located at the same path length into the tissue, with this distance now measured along the new optical path as determined by Snell's law. It is assumed that we know (1) the angle of incidence and (2) the indices of refraction for both the tissue and the medium through which the light is delivered. The net result is a change in the positions of the point sources, particularly a shift in the $x$ direction. Wang and Jacques' original presentation of obliqueincidence reflectometry, ${ }_{1}^{1}$ for which a Monte Carlo simulation and a least-squares fitting to this model were used, showed that the buried source is actually located at a depth (measured along the optical path) of

$$
1 /\left(0.35 \mu_{a}+\mu_{s}^{\prime}\right) \approx \mathrm{mfp}^{\prime} .
$$

Note that this expression is not exactly equal to 1 mfp' [Eq. (1)]. The factor of 0.35 has a significant effect for media with moderately high absorption relative to scattering. From the diffusion approximation to transport theory, it is known that

$$
3 D \approx 1 \mathrm{mfp}^{\prime} .
$$

We suggest that the empirical result of approximation (2a) is actually a more accurate expression for the diffusion coefficient $D$ and define it accordingly:

$$
3 D=1 /\left(0.35 \mu_{a}+\mu_{s}^{\prime}\right) .
$$



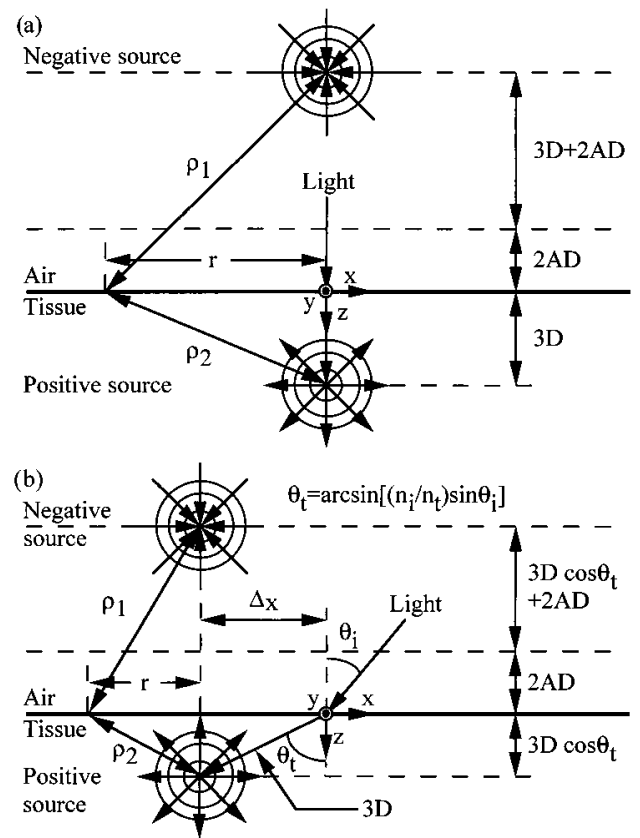

Fig. 2. (a) Positions of point sources in the diffusion theory model for normal incidence, (b) positions of point sources in the diffusion theory model for oblique incidence. The $y$ axis points off the page. $\rho_{1}$ and $\rho_{2}$ are the distances from the positive and the negative point sources, respectively, to the point of interest on the tissue surface at a radius $r$ from the axis of the sources. $\theta_{t}$ is determined from Snell's law (see Fig. 5 below).

We believe that for both normal and oblique incidence, the more accurate expression for the path length from the tissue surface to the positive point source is what we have defined as $3 D$ [Eq. (2c)] rather than $1 \mathrm{mfp}^{\prime}$ [Eq. (1)]. These two cases are diagrammed in Figs. 2(a) and 2(b).

The diffuse reflectance profile for oblique incidence is centered about the position of the point sources, so we can measure the shift $\Delta x$ by finding the center of diffuse reflectance relative to the light entry point. As is the case for normal incidence, the diffusion theory model, when shifted by $\Delta x$, also agrees with Monte Carlo results outside of 1-2 $\mathrm{mfp}^{\prime}$ from the center of diffuse reflectance, which, it is important to reiterate, is no longer at the point of entry [Fig. 1(b)].

The two-source model, with a depth of $3 D$ instead of $1 \mathrm{mfp}^{\prime}$, gives the following expression 8 :

$$
\begin{aligned}
R(r)= & 3 D\left(\mu_{\mathrm{eff}}+\frac{1}{\rho_{1}}\right) \frac{\exp \left(-\rho_{1} \mu_{\mathrm{eff}}\right)}{\rho_{1}^{2}}+(3 D+4 A D) \\
& \times\left(\mu_{\mathrm{eff}}+\frac{1}{\rho_{2}}\right) \frac{\exp \left(-\rho_{2} \mu_{\mathrm{eff}}\right)}{\rho_{2}^{2}},
\end{aligned}
$$

which can be scaled arbitrarily to fit a relative reflectance profile that is not in absolute units. $\mu_{\mathrm{eff}}$, the effective attenuation coefficient, is defined as

$$
\mu_{\mathrm{eff}}=\left(\mu_{a} / D\right)^{1 / 2} \text {. }
$$

$\rho_{1}$ and $\rho_{2}$ are the distances from the two sources to the point of interest (the point of light collection; see Fig.
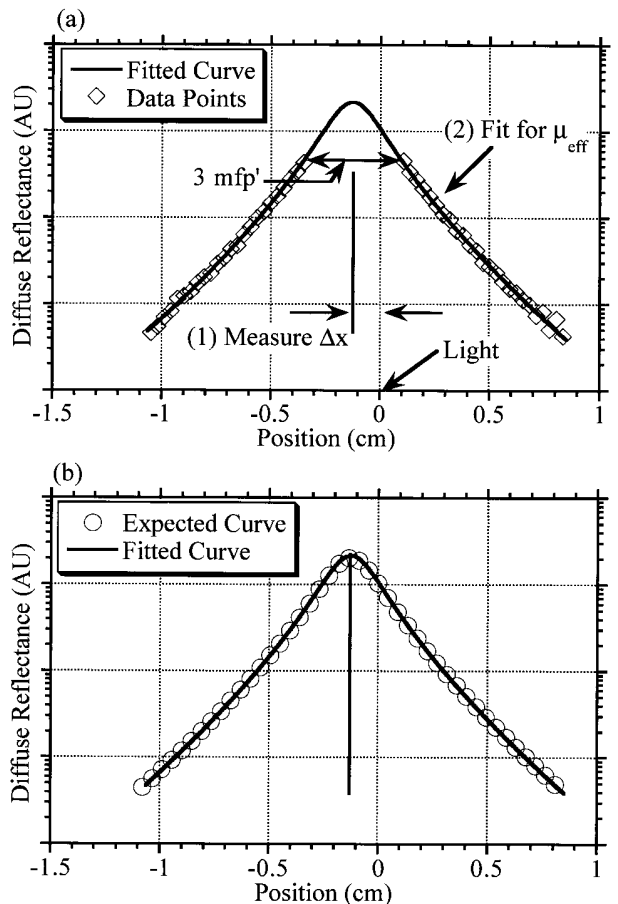

Fig. 3. (a) Sample of Monte Carlo simulated data after the removal of noise far from the point of entry as well as data within 1.5 $\mathrm{mfp}^{\prime}$ of the center of diffuse reflectance. The curve shown is the fitted result generated by our algorithm. To emphasize how well these curves agree with what we expected, in (b) we have plotted just the expected and the fitted curves without the simulation data. This particular simulation used $\left(\mu_{s}^{\prime}, \mu_{a}\right)=\left(6 \mathrm{~cm}^{-1}, 0.6 \mathrm{~cm}^{-1}\right)$.

2 ), and the boundary condition is included in the term $A^{9}$ :

$$
A=\left(1+r_{i}\right) /\left(1-r_{i}\right)
$$

where

$$
r_{i}=-1.440 n_{\mathrm{rel}}^{-2}+0.710 n_{\mathrm{rel}}^{-1}+0.668+0.0636 n_{\mathrm{rel}},
$$

$n_{\text {rel }}=n_{\text {tissue }} / n_{\text {ambient }}$.

As can be seen in Fig. 2(b), the diffusion coefficient can be calculated from $\Delta x$ :

$$
D=\Delta x /\left(3 \sin \theta_{\text {tissue }}\right) .
$$

Our method is as follows [see Figs. 3(a) and 4(a)]:

(1) Sample the relative diffuse reflectance profile at known positions from the light entry point.

(2) Search for the center of diffuse reflectance by (a) interpolating between the data points taken several $\mathrm{mfp}^{\prime}$ from the source and (b) finding the apparent center of the symmetrical reflectance profile.

(3) Calculate $\Delta x$ and $D . \Delta x$ is the distance between the point of light incidence and the apparent center of diffuse reflectance. $D$ follows from Eq. (8).

(4) Calculate the positions in the $z$ direction of the buried (positive) source and the image (negative) source as depicted in Fig. 2(b). 

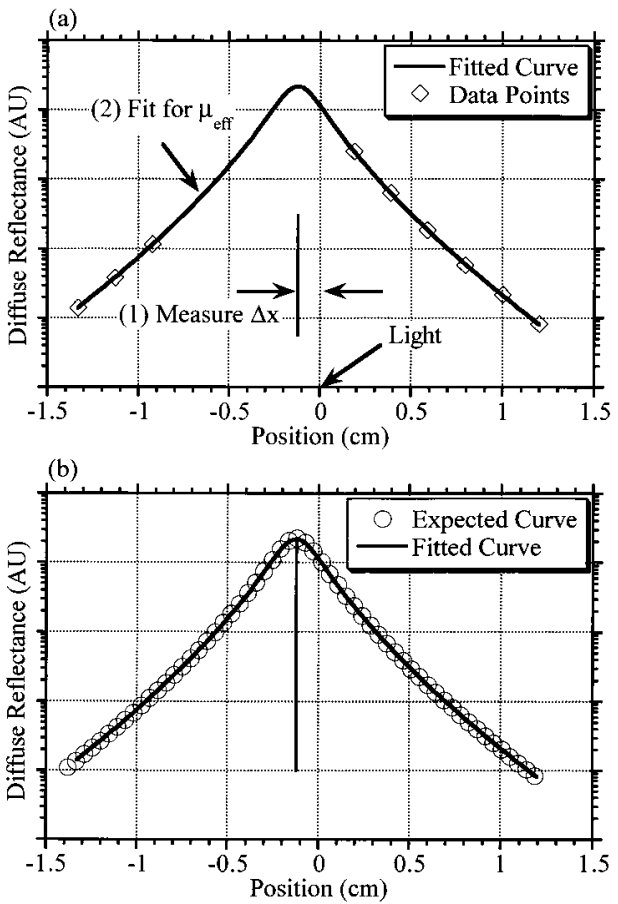

Fig. 4. (a) Sample of data taken with oblique-incidence fiber-optic probe. As in Fig. 3, the curve shown is the fitted result generated by our algorithm. In (b) we have plotted just the expected and the fitted curves without the data to reiterate how well they agree. This particular phantom had $\left(\mu_{s}^{\prime}, \mu_{a}\right)=\left(6 \mathrm{~cm}^{-1}, 0.6 \mathrm{~cm}^{-1}\right)$.

(5) Perform a nonlinear least-squares fit with the Levenberg-Marquardt method on Eq. (3) to determine $\mu_{\text {eff. }}{ }^{10}$

(6) Solve for $\mu_{a}$ and $\mu_{s}^{\prime}$ from the expressions

$$
\begin{aligned}
& \mu_{a}=D \mu_{\mathrm{eff}}^{2}, \\
& \mu_{s}^{\prime}=1 /(3 D)-0.35 \mu_{a} .
\end{aligned}
$$

\section{B. Monte Carlo Simulations}

The Monte Carlo simulations were run on a Sun Sparcstation 10.6,7,11 Over six simulations the optical properties were varied from 0.2 to $0.6 \mathrm{~cm}^{-1}$ for $\mu_{a}$ and from 4 to $10 \mathrm{~cm}^{-1}$ for $\mu_{s}^{\prime}$. The number of photon packets used varied from $6 \times 10^{6}$ to $10 \times 10^{6}$, depending on the optical properties. Light was launched from a refractive-index-matched ambient (to give a matched boundary condition, $n_{\text {rel }}=A=1$ ) into the tissue at an angle of $51^{\circ}$. This angle was chosen to match the refracted angle of transmission from our fiber-optic probe (see Subsection 2.C) and thus gave us the same optical path inside the turbid media for both the simulations and experiments.

The reflectance data were collected in a plane parallel to the plane of incidence, but offset by a small distance, $\Delta y$ (Fig. 5). The offset was also chosen to match the physical dimensions of the probe. Diffusely reflected photons were counted in bins extending $0.1 \mathrm{mfp}^{\prime}$ in $x$ and $0.03735 \mathrm{~cm}$ in $y$. Noisy data near $0.9 \mathrm{~cm}$ and farther from the point of incidence were removed. In addition, we also cut out any data

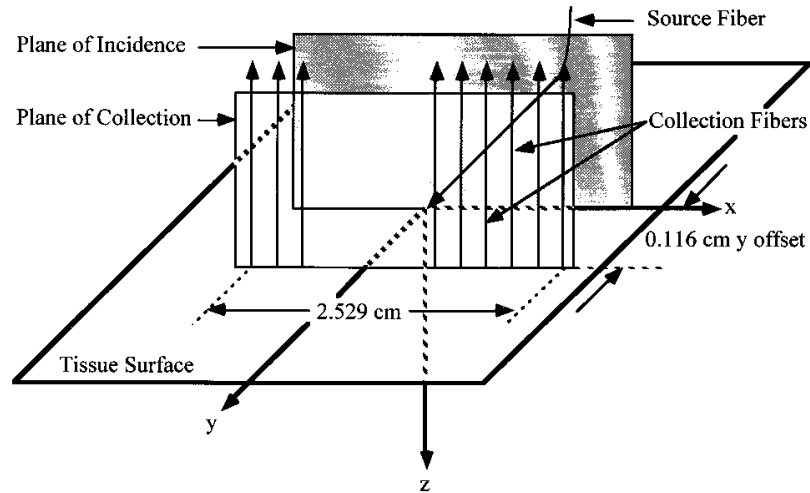

Fig. 5. Three-dimensional perspective view of tissue surface, coordinate axes, and positions of light delivery and collection. The arrows represent the fibers in the probe. The arrows pointing up are the collection fibers and the curved arrow is the source fiber.

within $1.5 \mathrm{mfp}^{\prime}$ of the center of diffuse reflectance. Finally, the simulated data was input to our fitting algorithm, which plots both the expected and the fitted curves based on diffusion theory. The expected curve was based on the optical properties defined for the simulated tissue. The program, which is implemented in C, also outputs relative errors for all the fitted parameters. See Fig. 3 and Section 4 for a discussion. The results of this procedure for all six sets of optical properties used are given in Subsection 3.A.

\section{Fiber-Optic Probe Experiments}

Our oblique-incidence optical fiber probe was made out of black Delrin and a 600- $\mu \mathrm{m}$-diameter, low-loss optical fiber. There was one source fiber, which was bent to an angle of $45^{\circ}$, and there are nine collection fibers, all aligned normally and offset from the plane of incidence by a small distance $\Delta y$ (Fig. 5). The tilt in the source fiber resulted in a transmitted angle (after refraction) of $51^{\circ}$, which is the same angle we used in the simulations. The fiber positions were measured with a resolution of $20 \mu \mathrm{m}$ from a digitized video image of the probe face. A 632.8-nm He-Ne laser was the light source, and an SMA-coupled photomultiplier tube measured the amount of light collected in each detection fiber. To approximate a matched boundary condition (no light reflected back from the tissue surface) we floated a piece of black, exposed x-ray film on top of each tissue phantom. The film had a small window through which to place the probe in contact with the phantoms. See Section 4 for a discussion.

Tissue phantoms with optical properties similar to those of biological tissue were prepared from water, Trypan Blue dye (an essentially pure absorber), and 900-nm-diameter polystyrene spheres (an essentially pure scatterer). We measured the absorption coefficient of the Trypan Blue stock solution with a spectrophotometer, calculated the scattering anisotropy of the spheres with Mie theory, ${ }^{12}$ and measured the scattering coefficient of the spheres by collimated transmission. ${ }^{13}$ The dilutions necessary to $\mathrm{mix}$ 
Table 1. Results from Monte Carlo Simulations

\begin{tabular}{|c|c|c|c|c|c|c|c|}
\hline \multirow{2}{*}{$\begin{array}{c}\text { Fitted } \\
\text { Parameter }\end{array}$} & \multirow[b]{2}{*}{ Values } & \multicolumn{6}{|c|}{ Expected Values of $\left(\mu_{s}^{\prime}, \mu_{a}\right)\left(\mathrm{cm}^{-1}, \mathrm{~cm}^{-1}\right)$} \\
\hline & & $(4,0.4)$ & $(6,0.2)$ & $(6,0.4)$ & $(6,0.6)$ & $(8,0.4)$ & $(10,0.4)$ \\
\hline \multirow[t]{3}{*}{$\Delta x\left(\mathrm{~cm}^{-1}\right)$} & Expected & 0.188 & 0.128 & 0.126 & 0.125 & 0.095 & 0.077 \\
\hline & Fitted & 0.188 & 0.130 & 0.128 & 0.125 & 0.098 & 0.077 \\
\hline & $\%$ Error & 0.4 & 1.7 & 1.5 & 0.1 & 2.5 & 0.8 \\
\hline \multirow[t]{3}{*}{$\mu_{\mathrm{eff}}\left(\mathrm{cm}^{-1}\right)$} & Expected & 2.229 & 1.908 & 2.714 & 3.343 & 3.125 & 3.488 \\
\hline & Fitted & 2.262 & 1.893 & 2.738 & 3.309 & 3.117 & 3.440 \\
\hline & $\%$ Error & 1.5 & -0.8 & 0.9 & -1.0 & -0.3 & -1.4 \\
\hline \multirow[t]{3}{*}{$\mu_{a}\left(\mathrm{~cm}^{-1}\right)$} & Expected & 0.4 & 0.2 & 0.4 & 0.6 & 0.4 & 0.4 \\
\hline & Fitted & 0.414 & 0.200 & 0.413 & 0.587 & 0.408 & 0.392 \\
\hline & $\%$ Error & 3.4 & 0.1 & 3.3 & -2.1 & 2.0 & -1.9 \\
\hline \multirow[t]{3}{*}{$\mu_{s}^{\prime}\left(\mathrm{cm}^{-1}\right)$} & Expected & 4.0 & 6.0 & 6.0 & 6.0 & 8.0 & 10.0 \\
\hline & Fitted & 3.980 & 5.897 & 5.906 & 6.009 & 7.798 & 9.919 \\
\hline & $\%$ Error & -0.5 & -1.7 & -1.6 & 0.2 & -2.5 & -0.8 \\
\hline
\end{tabular}

phantoms with the desired optical properties were calculated from the absorption and reduced scattering coefficients of the stock solutions. We made 10 phantoms with $\mu_{a}$ varying from 0.2 to $0.6 \mathrm{~cm}^{-1}$ and $\mu_{s}^{\prime}$ from 4 to $10 \mathrm{~cm}^{-1}$. Six of the 10 sets of optical properties were the same as those used in the simulations. Each phantom had a total volume of $500 \mathrm{ml}$, and they were contained in cylindrical buckets with a radius of $5 \mathrm{~cm}$ and a height of $8 \mathrm{~cm}$. The longest $1 / e$ penetration depth was approximately $0.5 \mathrm{~cm}\left[\right.$ for $\left(\mu_{s}^{\prime}, \mu_{a}\right)=(6$ $\left.\mathrm{cm}^{-1}, 0.183 \mathrm{~cm}^{-1}\right)$, which ensured a distance of 10 penetration depths to the sides of the bucket and 16 penetration depths to the bottom; hence our requirement for a semi-infinite medium [implicit in Eq. (3)] is fulfilled. To decrease the error in the expected values for each phantom, the absorber was diluted first, and an independent measurement of absorption was taken with the spectrophotometer before the addition of the scatterer. The expected phantom optical properties were thus derived from (1) an accurate, independent measurement of absorption, (2) the assumption that the scatterer was accurately diluted to the desired values, and (3) the assumption that there was no interaction between the absorber and the scatterer to change the final optical properties of the phantoms, once all the components were mixed.

The diffuse reflectance profile for each phantom was collected independently four times. The four phantoms with $\left(\mu_{s}^{\prime}, \mu_{a}\right)=\left(6 \mathrm{~cm}^{-1}, 0.374 \mathrm{~cm}^{-1}\right),(8$ $\left.\mathrm{cm}^{-1}, 0.386 \mathrm{~cm}^{-1}\right),\left(7 \mathrm{~cm}^{-1}, 0.178 \mathrm{~cm}^{-1}\right)$, and $\left(7 \mathrm{~cm}^{-1}\right.$, $0.589 \mathrm{~cm}^{-1}$ ) were used as calibration standards and the remaining six as test standards. The calibration standards were used to calculate the correction factors for the collection fibers, and the test standards were used as knowns against which to compare our measured optical properties. Because each fiber has a slightly different collection efficiency that is due to imperfections in polishing and differences in coupling efficiency to the detector, a calibration factor $k_{i}$ (where $i$ is the fiber number) is necessary for each collection fiber. We calculated the $k_{i}$ 's by making the measured curve match the expected curve for each calibration phantom (with an arbitrary scaling factor). We then calculated the average $k_{i}$ 's over the four calibration phantoms and used these average values as the calibration factors for the remainder of the experiment.

The raw data from each of the remaining six test standards were first corrected with the calibration factors, then input to the same fitting algorithm used in the simulations. See Fig. 4 for an example and Subsection 3.B for results.

\section{Results}

\section{A. Monte Carlo Results}

Table 1 contains the compiled results from six Monte Carlo simulations. Included are all fitted values, expected values, and their respective errors for both the directly fitted parameters $\Delta x$ and $\mu_{\text {eff }}$ and the final calculated values of $\mu_{a}$ and $\mu_{s}^{\prime}$. The majority of errors fell in the range of $-2 \%$ to $+2 \%$, with the largest errors of $3.3 \%$ and $3.4 \%$ in the determination of $\mu_{a}$.

\section{B. Experimental Results}

Table 2 contains the compiled results from the experiments with the fiber-optic probe. Four phantoms with optical properties $\left(\mu_{s}^{\prime}, \mu_{\alpha}\right)=\left(6 \mathrm{~cm}^{-1}, 0.374\right.$ $\left.\mathrm{cm}^{-1}\right),\left(8 \mathrm{~cm}^{-1}, 0.386 \mathrm{~cm}^{-1}\right),\left(7 \mathrm{~cm}^{-1}, 0.178 \mathrm{~cm}^{-1}\right)$ and $\left(7 \mathrm{~cm}^{-1}, 0.589 \mathrm{~cm}^{-1}\right)$ were used for calibration, as described in Subsection 2.B. The table gives the expected values, average fitted values, their associated errors, and the standard deviation for $\Delta x, \mu_{\mathrm{eff}}, \mu_{a}$, and $\mu_{s}^{\prime}$. The majority of errors easily fell in the range from $-5 \%$ to $+5 \%$, with the largest error of $-11.8 \%$ in the determination of $\mu_{a}$ for the phantom with a scattering of $4 \mathrm{~cm}^{-1}$ and absorption of $0.381 \mathrm{~cm}^{-1}$. The $\mathrm{mfp}^{\prime}$ for this phantom is $0.228 \mathrm{~cm}$, and after the expected shift in the center of diffuse reflectance of $0.188 \mathrm{~cm}$ is taken into account, it becomes clear that to eliminate data within $1.5 \mathrm{mfp}^{\prime}$ of the center, the source fiber can be no closer than $0.155 \mathrm{~cm}$ to the nearest collection fiber (the first one to the right of the source in Fig. 5). The distance from the source fiber to the nearest collection fiber is only $0.186 \mathrm{~cm} \pm 0.002$ $\mathrm{cm}$, and the collection fiber size is $600 \mu \mathrm{m}$. Thus the fiber tip actually extends from a position of 0.156 to $0.216 \mathrm{~cm}( \pm 0.002 \mathrm{~cm})$ and hence samples the reflec- 
Table 2. Results from Phantom Experiments

\begin{tabular}{|c|c|c|c|c|c|c|c|}
\hline \multirow{2}{*}{$\begin{array}{c}\text { Fitted } \\
\text { Parameter }\end{array}$} & \multirow[b]{2}{*}{ Values } & \multicolumn{6}{|c|}{ Expected Values of $\left(\mu_{s}^{\prime}, \mu_{a}\right)\left(\mathrm{cm}^{-1}, \mathrm{~cm}^{-1}\right)$} \\
\hline & & $(4,0.4)$ & $(6,0.2)$ & $(6,0.6)$ & $(8,0.2)$ & $(8,0.6)$ & $(10,0.4)$ \\
\hline \multirow[t]{4}{*}{$\Delta x\left(\mathrm{~cm}^{-1}\right)$} & Expected & 0.188 & 0.128 & 0.125 & 0.096 & 0.095 & 0.077 \\
\hline & Fitted & 0.182 & 0.130 & 0.123 & 0.098 & 0.095 & 0.080 \\
\hline & Std. deviation & 0.001 & 0.002 & 0.001 & 0.003 & 0.001 & 0.002 \\
\hline & $\%$ Error & -3.2 & 1.2 & -1.5 & 2.3 & 0.6 & 5.0 \\
\hline \multirow{4}{*}{$\mu_{\text {eff }}\left(\mathrm{cm}^{-1}\right)$} & Expected & 2.174 & 1.824 & 3.277 & 2.149 & 3.847 & 3.414 \\
\hline & Fitted & 2.091 & 1.817 & 3.199 & 2.148 & 3.873 & 3.415 \\
\hline & Std. deviation & 0.021 & 0.099 & 0.033 & 0.032 & 0.023 & 0.021 \\
\hline & $\%$ Error & $-4.5^{b}$ & -0.4 & -2.4 & -0.02 & 0.7 & 0.05 \\
\hline \multirow[t]{4}{*}{$\mu_{a}\left(\mathrm{~cm}^{-1}\right)$} & Expected & 0.381 & 0.183 & 0.577 & 0.191 & 0.601 & 0.383 \\
\hline & Fitted & 0.341 & 0.184 & 0.542 & 0.195 & 0.613 & 0.403 \\
\hline & Std. deviation & 0.008 & 0.023 & 0.016 & 0.010 & 0.012 & 0.012 \\
\hline & $\%$ Error & $-11.8^{b}$ & 0.8 & -6.1 & 2.3 & 2.0 & 5.1 \\
\hline \multirow{4}{*}{$\mu_{s}^{\prime}\left(\mathrm{cm}^{-1}\right)$} & Expected & 4.0 & 6.0 & 6.0 & 8.0 & 8.0 & 10.0 \\
\hline & Fitted & 4.155 & 5.929 & 6.107 & 7.822 & 7.948 & 9.515 \\
\hline & Std. deviation & 0.022 & 0.102 & 0.070 & 0.230 & 0.095 & 0.234 \\
\hline & $\%$ Error & 3.9 & -1.2 & 1.8 & -2.2 & -0.6 & -4.8 \\
\hline
\end{tabular}

${ }^{a}$ The sample size for each measurement was four.

${ }^{b}$ The error in $\mu_{\mathrm{eff}}$ drops to $-2.6 \%$ and the error in $\mu_{a}$ drops to $-8.2 \%$ when the fiber nearest the source is not included.

tance right at the edge of the region $1.5 \mathrm{mfp}^{\prime}$ from the center of diffuse reflectance. Removing this data point for this particular phantom decreased the average error in $\mu_{\text {eff }}$ from $-4.5 \%$ to $-2.6 \%$ and the average error in $\mu_{a}$ from $-11.8 \%$ to $-8.2 \%$. The errors for $\Delta x$ and $\mu_{s}^{\prime}$ remained unchanged.

\section{Discussion and Conclusions}

There are a number of points that should be clarified involving the collection and manipulation of data for both the Monte Carlo simulations and experiments. The first issue is that of the $y$ offset between the plane of incidence and the plane of collection (Fig. 5). Addition of this offset has one major effect: It increases the effective distances $\rho_{1}$ and $\rho_{2}$ from Eq. (3) between the virtual sources and the point of interest without increasing the width of the probe (size in $x$ dimension). The small increase in the thickness of the probe ( $y$ dimension) is negligible compared with the width. This is an advantage because it allows us to make more compact probes without compromising the requirement that data too close to the source be ignored. This probe shows better performance than our previous all-oblique design ${ }^{14}$ for two reasons: (1) The earlier design did not incorporate the additional $y$ offset for the collection fibers, and (2) the earlier design required each collection fiber to have a diffusing tip. Not only is it difficult to fabricate small, truly diffusing tips, but the diffuser also dramatically decreases light throughput.

Because any given probe will have predefined collection fiber positions, it is also important to realize that there is a physical limitation on the range of optical properties that can be accurately measured. The limiting factor is the distance from the source fiber to the nearest collection fiber. If the diffusion theory model is accurate only in the region farther than 1-2 $\mathrm{mfp}^{\prime}$ from the center of diffuse reflectance, then there is clearly a maximum value for the $\mathrm{mfp}^{\prime}$ of the tissue under investigation that can be measured correctly. The maximum value of $\mathrm{mfp}^{\prime}$ for our probe is approximately $0.23 \mathrm{~cm}$ (as described in Section 3), and the removal of data too close to the source improves the results significantly. In the simulations, all data within $1.5 \mathrm{mfp}^{\prime}$ of the expected center of diffuse reflectance were removed, and the fitted results were very good. This proves that, within these parameters, our fitting algorithm for reconstruction of the optical properties is valid. In an actual experiment, if the fitted results yield a mfp' of the order of the maximum measurable value of $0.23 \mathrm{~cm}$, then the results are prone to relatively high error and a larger probe should be used. At the other extreme, as the mfp' decreases, the only physical limitation is the sensitivity of the photodetector. The collection fibers farthest from the source might not collect a detectable amount of signal, and hence a smaller probe should be used.

In both the simulations and experiments, a matched boundary condition with $A=1$ was used to avoid the issue of what $A$ should be for a tissue-air interface. In the experiments, we approximated this condition by floating a black, exposed piece of $\mathrm{x}$-ray film on the phantom surfaces. The refractive index of the film $(n=1.5)$ matches that of the phantom $(n=1.33)$ reasonably well. The internal total diffuse reflectance coefficient (phantom-film interface) was approximately 0.03 , and the optical density of the film was over 4 OD (the limit of our spectrophotometer), so light reflecting off the film-air interface back into the phantom would be attenuated by at least 8 orders of magnitude. Thus the film is thick enough such that photons from inside the phantom do not effectively see the film-air interface. ${ }^{15}$

After the collection of raw data it is important to calibrate the probe for maximum accuracy. The pri- 
mary reason is to correct for variability in fibercollection efficiency. Because the fibers collect light in a fixed orientation relative to the inhomogeneous diffuse illumination from the source, the simplest way to calibrate is to mimic the situation of an actual measurement. It is possible to calibrate against a broad, flat-field beam instead, but this technique does not work as well because in the actual measurements the fiber tips are not exposed to true Lambertian sources. The flux is actually biased along the axis of the probe within which the fibers are fixed. In addition, it would be simpler in a clinical setting to calibrate periodically against a set of solid standards than an expanded single-mode beam. The calibration factors thus obtained theoretically should hold true for any optical properties being measured. These factors should also work for any wavelength of light, provided that there is no change in the relative collection efficiencies of the fibers from wavelength to wavelength. To decrease any small variations in the calibration factors that may be present, it is desirable to calibrate against several phantoms whose properties span the region of interest. This is not to say, though, that optical properties outside the region spanned by the calibration standards cannot be measured accurately. As mentioned above, the primary limit on what can be measured depends on the physical dimensions of any given probe relative to the optical properties of the unknown. Indeed, in our experiments we had little trouble taking measurements from phantoms with reduced scattering of 4 and $10 \mathrm{~cm}^{-1}$, whereas our calibration standards ranged from $\mu_{s}^{\prime}=6$ to $8 \mathrm{~cm}^{-1}$.

The final issue of discussion is that of error propagation. From Tables 1 and 2 it should be clear that the range of error in calculating $\mu_{a}$ is 2-3 times higher than that obtained for $\mu_{s}^{\prime}$ and the directly measured parameters of $\Delta x$ and $\mu_{\text {eff }}$. The reason is evident from Eq. (9). $\mu_{a}=D \mu_{\text {eff }}^{2}$, so the error in $\mu_{a}$ is of the order of the error in $D$ (or $\Delta x$, to which $D$ is proportional) plus twice the error in $\mu_{\mathrm{eff}}$. This is derived as follows:

$$
\begin{aligned}
\mu_{a} & =D \mu_{\mathrm{eff}}^{2}, \\
\ln \left(\mu_{a}\right) & =\ln (D)+2 \ln \left(\mu_{\mathrm{eff}}\right), \\
\Delta \mu_{a} / \mu_{a} & \approx(\Delta D / D)+2\left(\Delta \mu_{\mathrm{eff}} / \mu_{\mathrm{eff}}\right) .
\end{aligned}
$$

In contrast, the error in $\mu_{s}^{\prime}$ is of the order of the error in $D$ (or $\Delta x$ ), particularly for tissues with high albedo. This follows similarly from Eq. (10).

In conclusion, we have developed a method for quickly, cheaply, and noninvasively measuring the absorption and reduced scattering coefficients of turbid media with an oblique-incidence fiber-optic probe. The fundamental principle is that the shift in the center of diffuse reflectance from the light entry point gives us a measure of the diffusion length, and a curve fit to the diffusion theory model gives us a measure of the effective attenuation coefficient. $\mu_{\text {eff }}$ primarily determines the slope of the falloff of diffuse reflectance. Thus, with only a relative measurement of the diffuse reflectance profile, we can measure two parameters and hence deduce both optical properties, $\mu_{a}$ and $\mu_{s}^{\prime}$. Ongoing work involves using our probe for simultaneous multiwavelength measurements. With a white-light source, a spectrograph for spectral expansion, and a CCD camera, an image of spatially and spectrally resolved diffuse reflectance can be recorded and processed to yield efficiently the optical properties as a function of wavelength. ${ }^{16}$ It is with these multispectral measurements that the greatest advantage of oblique-incidence fiber-optic reflectometry becomes apparent. From spectrally resolved measurements of the broadband light collected by each detection fiber, the optical properties at any given wavelength can be reconstructed from the relative diffuse reflectance profile at that wavelength. The fitted properties will be the same regardless of the amount of source power delivered at that particular wavelength. Similarly, the spectral responsivity of the detector is also irrelevant. Thus, in contrast to methods that rely on spatially resolved measurements of diffuse reflectance from a normally incident light beam, with oblique incidence both absorption and scattering spectra can be obtained without any additional calibration. ${ }^{15,17}$

This project was sponsored in part by The Whitaker Foundation, U.S. National Institutes of Health grants R29 CA68562 and R29 HL45045, and the U.S. Department of Energy grant DE-FG05-91ER61226.

\section{References}

1. L.-H. Wang and S. L. Jacques, "Use of a laser beam with an oblique angle of incidence to measure the reduced scattering coefficient of a turbid medium," Appl. Opt. 34, 2362-2366 (1995).

2. J. W. Pickering, S. A. Prahl, N. Vanwieringen, J. F. Beek, H. J. C. M. Sterenborg, and M. J. C. van Gemert, "Doubleintegrating-sphere system for measuring the optical properties of tissue," Appl. Opt. 32, 399-410 (1993).

3. S. L. Jacques, A. Gutsche, J. A. Schwartz, L.-H. Wang, and F. K. Tittel, "Video reflectometry to specify optical properties of tissue in vivo," in Medical Optical Tomography: Functional Imaging and Monitoring, Vol. IS11 of SPIE Institute Series (Society of Photo-Optical Instrumentation Engineers, Bellingham, Wash. 1993), pp. 211-226.

4. A. Kienle, L. Lilge, M. S. Patterson, R. Hibst, R. Steiner, and B. C. Wilson, "Spatially resolved absolute diffuse reflectance measurements for noninvasive determination of the optical scattering and absorption coefficients of biological tissue," Appl. Opt. 35, 2304-2314 (1996).

5. T. J. Farrell, B. C. Wilson, and M. S. Patterson, "The use of a neural network to determine tissue optical properties from spatially resolved diffuse reflectance measurements," Phys. Med. Biol. 37, 2281-2286 (1992).

6. L.-H. Wang, S. L. Jacques, and L.-Q. Zheng, "MCML-Monte Carlo modeling of photon transport in multi-layered tissues," Comput. Methods Programs Biomed. 47, 131-146 (1995).

7. L.-H. Wang and S. L. Jacques, Monte Carlo Modeling of Light Transport in Multi-layered Tissues in Standard C (University of Texas M. D. Anderson Cancer Center, Houston, Texas, 1992). Note: available through e-mail to LWANG@tamu.edu.

8. T. J. Farrell, M. S. Patterson, and B. C. Wilson, "A diffusion theory model of spatially resolved, steady-state diffuse reflec- 
tance for the non-invasive determination of tissue optical properties in vivo," Med. Phys. 19, 879-888 (1992).

9. R. A. J. Groenhius, J. J. T. Bosch, and H. A. Ferwerdo, "Scattering and absorption of turbid materials determined from reflectance measurements. 1: theory," Appl. Opt. 22, 24562462 (1983).

10. W. H. Press, B. P. Flannery, S. A. Teukolsky, and W. T. Vetterlin, Numerical Recipes in $C$, 2nd ed. (Cambridge U. Press, Cambridge, UK, 1992), Section 15.5.

11. L.-H. Wang and S. L. Jacques, "Animated simulation of light transport in tissues," in Laser-Tissue Interaction V, S. L. Jacques, ed., Proc. SPIE 2134A, 247-254 (1994).

12. L.-H. Wang and S. L. Jacques, MIESPHR Program: Mie Theory for Scattering Spherical Particles (University of Texas M. D. Anderson Cancer Center, Houston, Texas, 1995). Note: available through anonymous ftp to laser.mda.uth.tmc.edu (129.106.60.92).

13. L.-H. Wang and S. L. Jacques, "Error estimation of measuring total interaction coefficients of turbid media using collimated light transmission,” Phys. Med. Biol. 39, 2349-2354 (1994).
14. L.-H. Wang, S.-P Lin, S. L. Jacques, F. K. Tittel, J. Harder, J. Jancarik, B. Mammini, W. Small, and L. Da Silva, "Obliqueincidence reflectometry: one relative profile measurement of diffuse reflectance yields two optical parameters," in Optical Biopsies, R. Cubeddu, S. R. Mordon, and K. Svanberg, eds., Proc. SPIE 2627, 165-175 (1995).

15. S.-P. Lin, "Oblique-incidence fiber-optic reflectometry for measuring absorption and scattering in turbid media," M.S. thesis (Rice University, Houston, Texas, 1996).

16. T. J. Farrell, M. S. Patterson, J. E. Hayward, B. C. Wilson, and E. R. Beck, "Charge-coupled device and neural-network-based instrument for the noninvasive determination of tissue optical properties in vivo," in Advances in Laser and Light Spectroscopy to Diagnose Cancer and Other Diseases, R. R. Alfano, ed., Proc. SPIE 2135, 117-128 (1994).

17. S.-P. Lin, L.-H. Wang, S. L. Jacques, and F. K. Tittel, "Measurement of absorption and scattering spectra with oblique incidence reflectometry," in Advances in Optical Imaging and Photon Migration, OSA Technical Digest Series (Optical Society of America, Washington, D.C., 1996). 